\title{
The double-sided ultrasonic beam displacement
}

\author{
Nico F. Declercq ${ }^{\text {a) }}$ and Joris Degrieck \\ Soete Laboratory, Department of Mechanical Construction and Production, Ghent University, \\ Sint Pietersnieuwstraat 41, 9000 Gent, Belgium \\ Oswald Leroy \\ Interdisciplinary Research Center, Katholieke Universiteit Leuven Campus Kortrijk, E. Sabbelaan 53, 8500 \\ Kortrijk, Belgium
}

(Received 25 February 2004; accepted 1 September 2004)

It is a well-established idea in optics as well as in ultrasonics that a bounded Gaussian beam, when reflected from an interface, can be displaced in the forward or in the backward direction, depending on the propagation direction of leaky waves that are generated by the incident beam. Such a displacement is often accompanied by the so-called Schoch effect characterized by a null strip in between a specular and a nonspecular reflected beam, and a trailing field that is much further displaced. The current letter shows experimentally and numerically that a simultaneous forward and backward displacement is possible accompanied by two null strips and being only the result of forward propagating Lamb waves. (c) 2004 American Institute of Physics. [DOI: $10.1063 / 1.1812363$ ]

In optics, the Goos-Hänchen theory predicts a lateral displacement of a light beam that is internally reflected from a dielectric interface. ${ }^{1}$ This phenomenon appears when incident from an optically denser medium at an angle close to the critical angle, resulting in the transfer of a portion of the energy into the rarer medium by means of excitation of an electromagnetic field that travels along the interface. This energy leaks back into the denser medium and becomes part of the reflected beam exhibiting a lateral displacement that appears as a forward beam shift. This was studied by Tamir and Bertoni. ${ }^{2}$ The early experiments of Schoch $^{3-5}$ applying the acoustic analog of the Goos-Hänchen effect for an acoustic beam reflected from a liquid-solid interface showed a forward lateral displacement of the reflected ultrasonic beam. It has been shown before ${ }^{6-8}$ that a backward displacement of an ultrasonic beam is also possible when backward propagating surface waves are stimulated on periodically rough surfaces. The expression "null strip" was mentioned in Neubauer and Dragonet. ${ }^{9}$ The effect consisting of both a forward and a backward displacement on a smooth interface, accompanied by two null strips, has never been observed and has certainly never been published before. The experiments in this letter are performed by means of a Schlieren experimental setup ${ }^{10}$ and the liquid/solid interfaces are smooth.

There is an unwritten law that whenever leaky Rayleigh waves or leaky Lamb waves are generated by means of an incident bounded beam, the Schoch effect occurs and vice versa. As an example, a typical Schoch effect is shown in Fig. 1, where sound is incident on a thin aluminum plate under a Lamb wave angle. It is seen that there is no backward displacement of the reflected beam. What is visible, is a forward displaced (nonspecular) sound lobe separated from the main (specular) reflected sound beam by a null strip. This is because the nonspecular lobe is out of phase with the specular sound beam. The null strip is the result of phase canceling. A trailing field at larger distances is also visible.

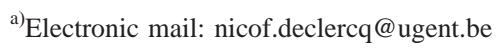

We have performed experiments on glass plates. The longitudinal sound velocity in glass is $5660 \mathrm{~m} / \mathrm{s}$, whereas the transversal sound velocity is $3520 \mathrm{~m} / \mathrm{s}$. The density is $2500 \mathrm{~kg} / \mathrm{m}^{3}$. Water is characterized by a longitudinal sound velocity of $1480 \mathrm{~m} / \mathrm{s}$ and a density of $1000 \mathrm{~kg} / \mathrm{m}^{3}$. The well-expected Schoch phenomenon was visible at certain angles depending on the frequency that was used and depending on the thickness of the plate. Nevertheless, for the combination of a plate thickness of $1.23 \mathrm{~mm}$ and a frequency of $3 \mathrm{MHz}$, a completely unexpected phenomenon appeared. The physical beam width was $1 \mathrm{~cm}$. The result can be seen in Fig. 2. The glass plate is indicated by means of white dashed lines. The white arrow denotes the direction of incidence. There is a forward displaced lobe and also a backward displaced lobe. Both lobes are separated from the specular lobe by means of a null strip. If the left lobe was not backward displaced and was hence the specular lobe, the effect could

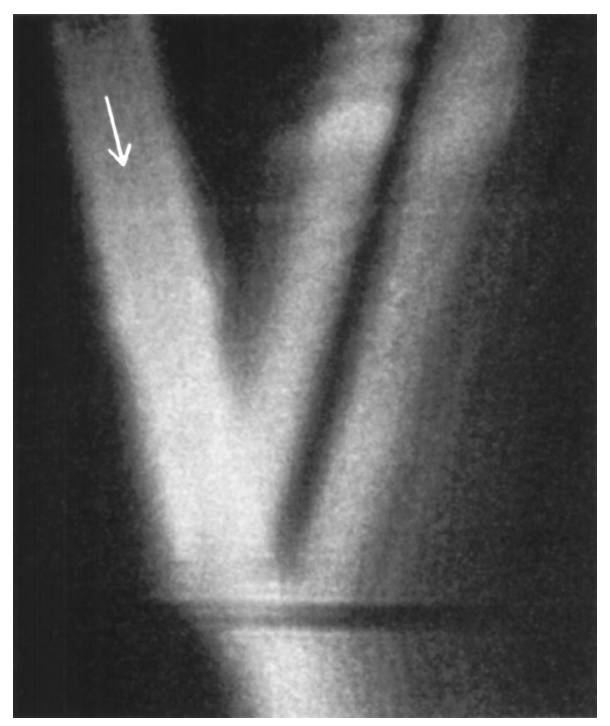

FIG. 1. The typical Schoch effect of a bounded ultrasonic beam on a thin aluminum plate. Only a forward displacement is visible accompanied by a trailing field. 


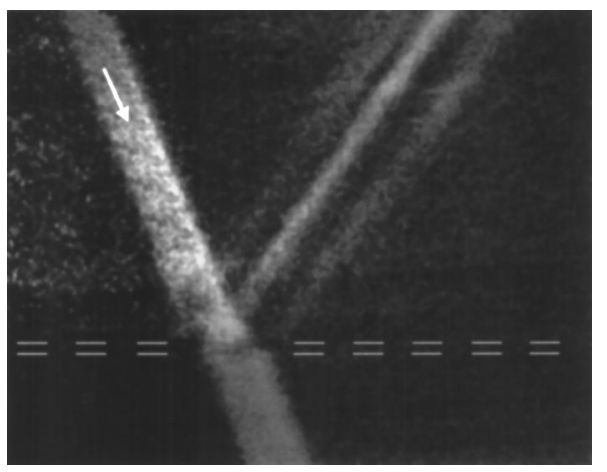

FIG. 2. The double-sided beam deformation. A backward and a forward displaced beam are visible together with the central specular reflected beam.

have been regarded as a very strong trailing field. However it is clear that the first lobe is not a specular lobe but is indeed backward displaced. In order to make sure that the effect was not caused by edge effects of the plate that was used, we have studied different positions of incidence and we have also studied the effect of smooth edges and rough edges. No difference was visible except when incidence occurred so close to the edge that the forward displaced sound beam "touched" or even surpassed the edge.

Simulations were performed by means of a decomposition of the incident bounded beam into plane waves in accordance with the Fourier transform. Continuity of normal stress and normal particle displacement was considered ${ }^{11}$ along the water-glass interfaces of the plate. The reflected profile was studied along the interface water/upper side of the plate. The same parameters were used as in the experiments. The reflected beam profile as a function of the angle of incidence can be seen in Fig. 3. It is seen that at $18.29^{\circ}$ and at $13.91^{\circ}$ there is a regular Schoch effect. Nevertheless, at $28.35^{\circ}$ there are two null zones separating three reflected beams. This is the double-sided beam deformation that corresponds to Fig. 2. The mentioned angles correspond to the dispersion curves of Fig. 4, denoting the pole position of the reflection coefficient for pure harmonic plane waves. It is seen that the double beam deformation effect occurs at the

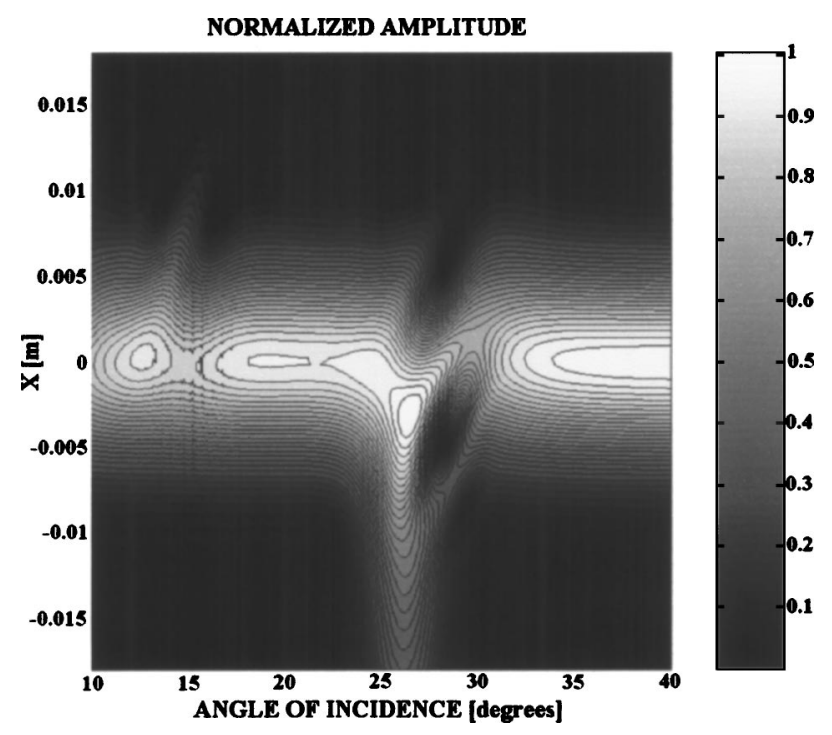

FIG. 3. Simulation (filled contour plot) of the reflected beam profile as a function of the angle of incidence. Negative positions correspond with backward positions. At $28.35^{\circ}$ the situation of Fig. 2 can be seen.

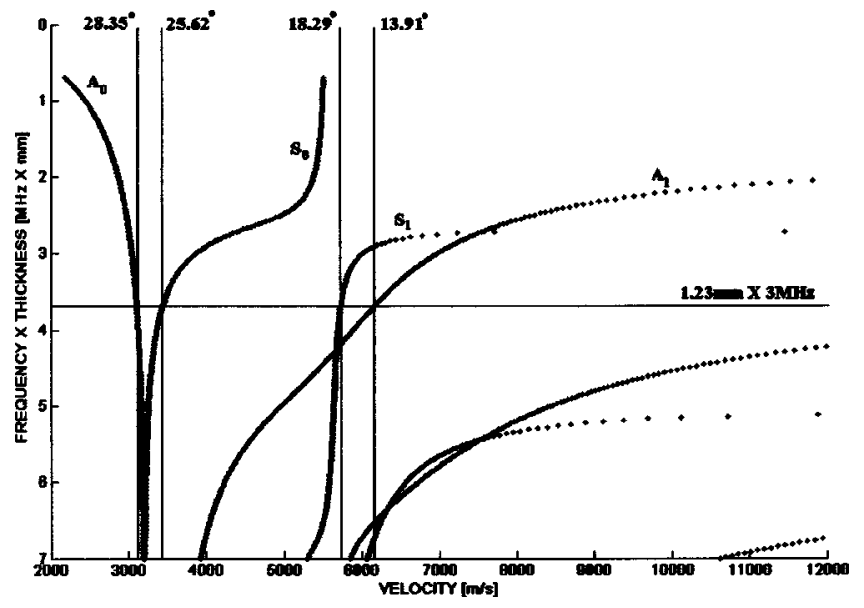

FIG. 4. The dispersion curves of a glass plate. The horizontal line corresponds to the experiments reported here. The vertical lines correspond to the angles of incidence of $28.38^{\circ}, 25.62^{\circ}, 18.29^{\circ}$, and $13.91^{\circ}$.

angle that stimulates the $A_{0}$ Lamb mode. The Schoch effect is not visible for the $S_{0}$ stimulating angle. The calculated profile in amplitude and in phase corresponding to the angle of incidence of $28.35^{\circ}$ is shown in Fig. 5, where it is seen that the left and the right lobes are out of phase with the central specular reflected lobe. A backward propagating sound field could be characterized by a negative group velocity. However, according to the dispersion curves in Fig. 4 and according to knowledge obtained from Ref. 12, this is never the case and hence there is no backward propagating sound field present. Therefore the effect is the result of a (complicated) interaction between the amplitudes and phases of the plane waves that are the building blocks of the incident bounded beam and the generated Lamb waves.

As a conclusion, it is shown experimentally and numerically that double beam deformations are possible on smooth plates swamped in water. It is also shown that this effect, in the example studied here, is not caused by backward propagating sound in the plate.

Work supported by "The Flemish Institute for the Promotion of the Scientific and Technological Research in Industry (I.W.T.)."
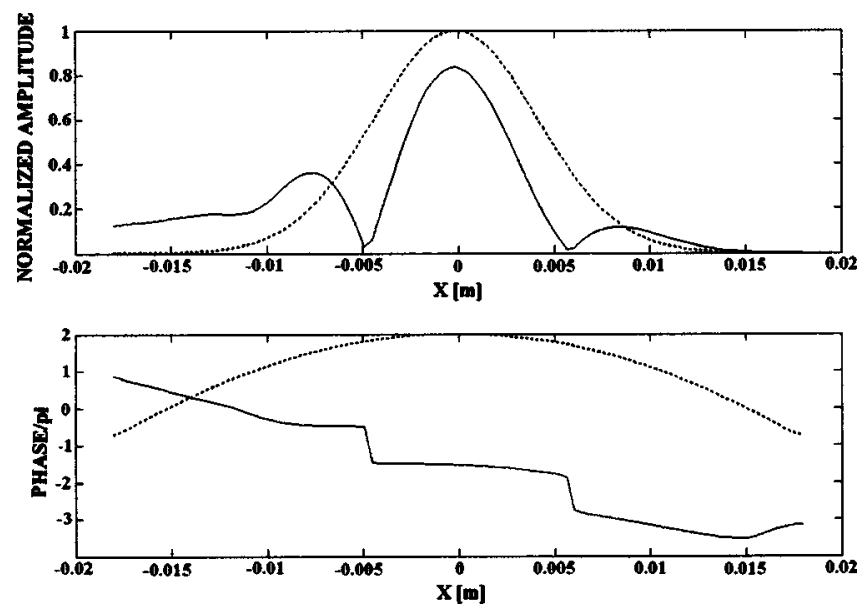

FIG. 5. Simulation of Fig. 2, i.e., incidence at $28.35^{\circ}$. Dotted curve: incident beam profile, solid curve: reflected beam profile. Note that the forward and the backward displaced beams are out of phase with the specular central lobe. 
${ }^{1}$ Von F. Goos and H. Hänchen, Ann. Phys. (Leipzig) 6, 333 (1947).

${ }^{2}$ T. Tamir and H. L. Bertoni, J. Acoust. Soc. Am. 61, 1397 (1971).

${ }^{3}$ A. Schoch, Nuovo Cimento, Suppl. 7, 302 (1950).

${ }^{4}$ A. Schoch, Acustica 2, 1 (1952).

${ }^{5}$ A. Schoch, Acustica 2, 18 (1952).

${ }^{6}$ M. A. Breazeale and Michael Torbett, Appl. Phys. Lett. 29, 456 (1976).

${ }^{7}$ N. F. Declercq, J. Degrieck, R. Briers, and O. Leroy, J. Acoust. Soc. Am. 112, 2414 (2002).
${ }^{8}$ N. F. Declercq, J. Degrieck, R. Briers, and O. Leroy, Appl. Phys. Lett. 82, 2533 (2003).

${ }^{9}$ W. G. Neubauer and L. R. Dragonet, J. Appl. Phys. 45, 618 (1974).

${ }^{10}$ M. A. Breazeale, J. Opt. A, Pure Appl. Opt. 3, S1 (2001).

${ }^{11}$ A. H. Naefeh, Wave Propagaton in Layered Anisotropic Media with Applications to Composites (North-Holland, Amsterdam, 1995).

${ }^{12}$ M. F. Werby and H. Überall, J. Acoust. Soc. Am. 111, 2686 (2002). 
Applied Physics Letters is copyrighted by the American Institute of Physics (AIP). Redistribution of journal material is subject to the AIP online journal license and/or AIP copyright. For more information, see http://ojps.aip.org/aplo/aplcr.jsp 